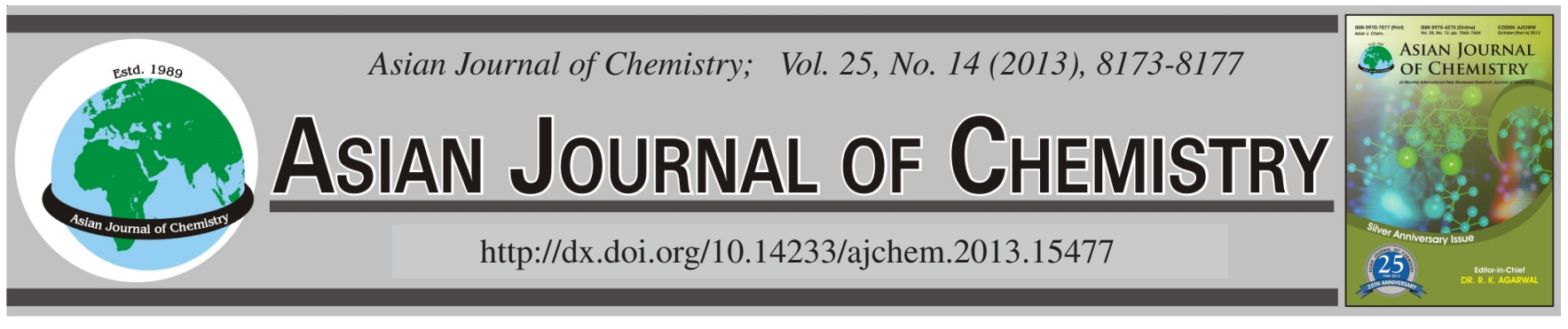

\title{
Heavy Atom Effect on the Room Temperature Chemiluminescent Phosphorescence of the Tricyclic 1,2-Dioxetanes
}

\author{
Chung-Wen Sun ${ }^{1}$, Shun-Chi Chen ${ }^{2, *}$ and TAI-Shan Fang ${ }^{1, *}$
}

${ }^{1}$ Department of Chemistry, National Taiwan Normal University, Taipei 116, Taiwan

${ }^{2}$ Department of Chemical Engineering, Ming Chi University of Technology, Taishan, Taipei 243, Taiwan

*Corresponding author: E-mail: scchemts@ntnu.edu.tw; scchen@mail.mcut.edu.tw

\begin{abstract}
1,2-Dioxetane tricyclic compound (1a) and 5-halo substituted of 1,2-dioxetane tricyclic compounds (2a, 3a) were synthesized from 1,4dioxin acenaphthylene compound (1) and 5-halo substituted 1,4-dioxin acenaphthylene compounds (2, 3), by reacting with singletoxygen $\left({ }^{1} \mathrm{O}_{2}\right)$ at $238 \mathrm{~K}$ and their chemiluminescent efficiency both in fluorescence and phosphorescence was studied while upon heating to temperatures between $313-353 \mathrm{~K}$ in dichloromethane. The chemiluminescent phosphorescence can be detected along with the chemiluminescent fluorescence when compounds $(\mathbf{2 a}, \mathbf{3 a})$ are decomposed thermally to the corresponding naphthalene diesters (2b, 3b). Experimental evidences for formation of the dioxetanes 1a-3a are the observed chemiluminescence spectra which are consistent with the photoluminescence spectra of the excited compounds $\mathbf{1 b}-\mathbf{3 b}$. Temperature-dependent kinetic measurements reveal an increase of room temperature chemiluminescent phosphorescence efficiency from triplet chemiexcitation processes due to the internal heavy atom effect of compound 3a.
\end{abstract}

Key Words: Chemiluminescence, Fluorescence, Phosphorescence, Dioxetane, Heavy atom effect.

\section{INTRODUCTION}

Photoluminescence is a type of optical spectroscopy in which a photoluminescent molecule is promoted to an electronically excited state by absorption of incident radiation. Chemiluminescence is a type of optical spectroscopy in which a chemiluminescent molecule is generated to an electronically excited state by chemiexcitation reactions. There are few reports about the room temperature chemiluminescent fluorescence and phosphorescence. For example, room temperature luminescence is rarely observed from the thermal decomposition of the simple 1,2-dioxetanes which produce excited carbonyl compounds. The energy in the form of light is released from 1,2-dioxetanes due to a chemiexcitation reaction during thermal decomposition. A simple example is the thermal decomposition of tetramethyl dioxetane (TMDO). In the TMDO structure, the $\mathrm{C}-\mathrm{O}-\mathrm{O}-\mathrm{C}$ is a twisted four-membered ring peroxide with high strain energy, which liberates much energy during thermal decomposition into two excited carbonyl fragments (singlet and triplet excited acetone) and then return to the ground state by chemiluminescence (Scheme-I) ${ }^{1-5}$.

The molecular structure of TMDO was used as a base template to design and synthesize compounds (1a-3a). The structure of the 1,2-dioxetane tricyclic compounds (1a-3a) are comprised of three parts. These are the 1,2-dioxetane ring (main structure), the electron-donor dialkoxy activator (tetramethyl 1,4-dioxin ring) on one side and the lumiphor (5heavy atom substituted acenaphtho moiety) on the other side (Scheme-II). The chloro- and bromo- substituents on the lumiphor are incorporated to study the internal heavy atom effect, which is an important factor for the room temperature

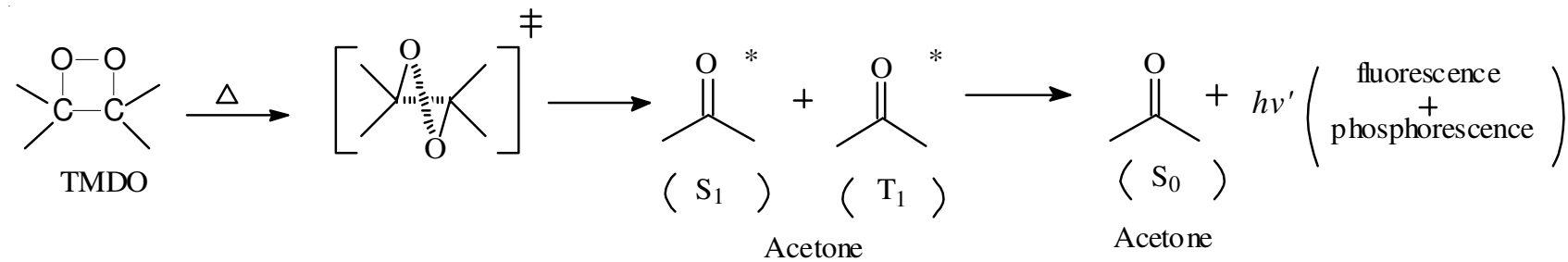

Scheme-I 


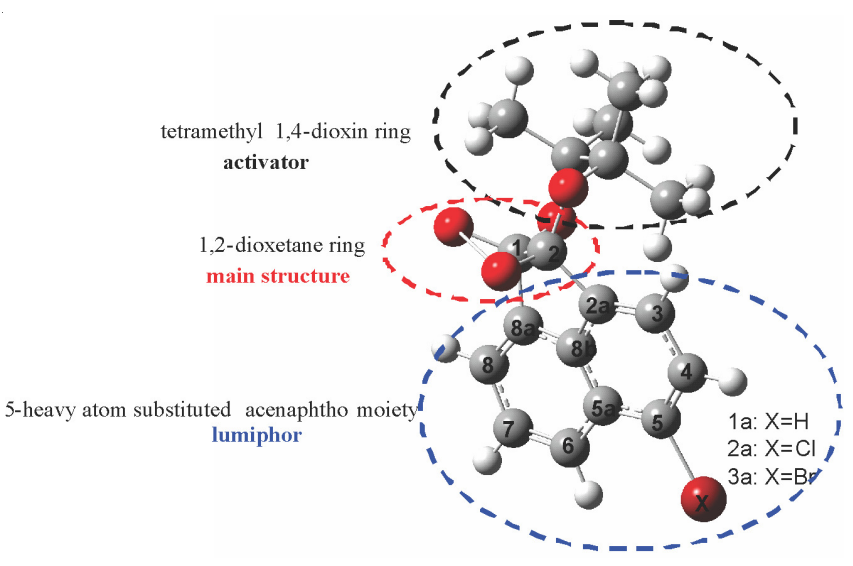

Scheme-II

chemiluminescent phosphorescence (RTCP) detection of chemiluminescent molecule by experimental measurements.

It is well known that the 1,2-dioxetane ring with its high strain energy undergoes simultaneous cleavage of both the $\mathrm{O}-\mathrm{O}$ and $\mathrm{C}-\mathrm{C}$ bonds during thermal decomposition into two excited intramolecular carbonyl fragments (singlet and triplet excited intramolecular naphthalene diester compounds $\mathbf{1} \mathbf{b}^{*}$ $\mathbf{3} \mathbf{b}^{*}$ ), which return to the ground state by chemiluminescent fluorescence $(\mathrm{CF})$ and chemiluminescent phosphorescence (CP), respectively ${ }^{6-9}$.

While it is difficult to detect the room temperature phosphorescence from compounds (1)-3b) under nondegassed condition, because of quenching by triplet-oxygen $\left({ }^{3} \mathrm{O}_{2}\right)$, the room temperature chemiluminescent phosphore-scence of compounds (1a-3a) is detected more easily, because the room temperature chemiluminescent phosphorescence emission derives from the chemiexcitation reactions of 1,2-dioxetanes. It is well known that the internal heavy atom effect can enhance the phosphorescence. This paper describes the room temperature chemiluminescent phosphorescence of 1,2-dioxetane tricyclic compound with a 5-heavy atom substituent in the acenaphtho moiety.

\section{EXPERIMENTAL}

1,4-Dioxin acenaphthylene, compounds (1-3): Acenaphthenequinone (ANQ) was obtained from Aldrich. The 5chloro acenaphthenequinone was synthesized from acenaphthenequinone and $\mathrm{N}$-chlorosuccinimide (NCS) in concentrated sulfuric acid. The reaction mixture was refluxed for $2 \mathrm{~h}$. The 5-bromo acenaphthenequinone was synthesized from acenaphthenequinone and bromine liquid. The reaction mixture was refluxed for $2 \mathrm{~h}^{10,11}$ (Scheme-III part A). The compounds (1-3) were synthesized by the photo-cycloaddition reaction of acenaphthenequinone (ANQ) with tetramethylethylene (TME) in benzene ${ }^{12}$ (Scheme-III part B).

1,2-Dioxetane tricyclic compounds (1a-3a): Singletoxygen oxidation of the 1,4-dioxin compounds (1-3) to produce the 1,2-dioxetane tricyclic compounds (1a-3a) was carried out using the ozone-triphenyl phosphite procedure given in ref. ${ }^{3}$. Dioxetane adducts (1a-3a) were isolated by ice-chilled $n$-hexane extraction from the low temperature synthesis of ozonized triphenyl phosphite [Scheme-III part C(1)].
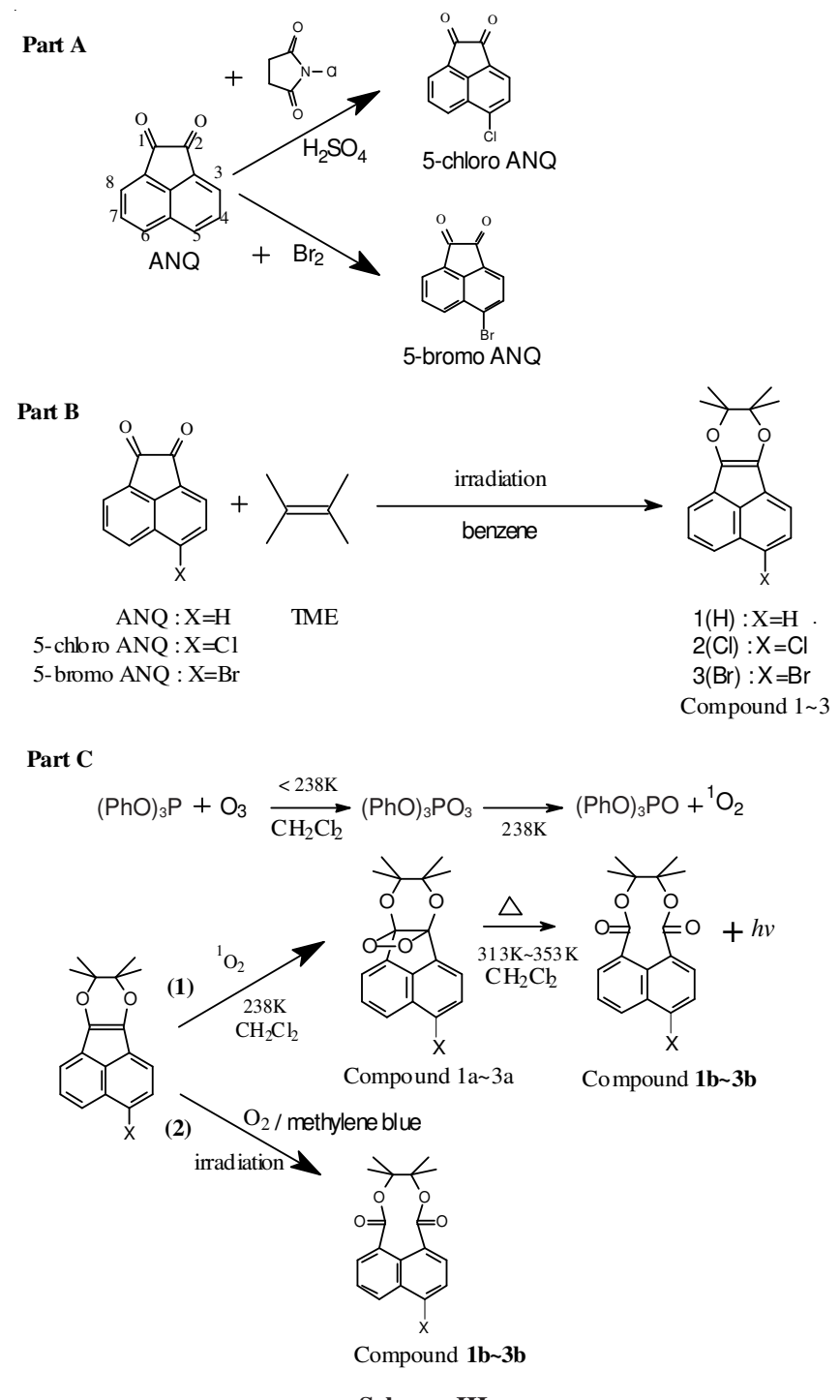

Naphthalene diester compounds (1b-3b): There are two methods to synthesize the naphthalene diester compounds (1b3b). (1) The compounds (1a-3a) were decomposed thermally to the naphthalene diester compounds (1b-3b) (Scheme-III part C(1)). (2) The compounds (1-3) in benzene solution were irradiated in the presence of photosensitizer methylene blue and purged oxygen [Scheme-III part C(2)].

Measurements: The UV/visible absorption spectra were measured on a Hewlett-Packard diode array spectrophotometer. The steady-state emission spectra were obtained using a Cary Eclipsed Spectrofluorimeter equipped with a temperature controller. Luminescence lifetimes were measured by a microsecond flash lamp Luminescence Spectrofluorimeter (Cary Eclipse) in a temperature range of 313-353 K. For the temperature-dependent kinetic measurements, the temperature of the sample solution were controlled to within $\pm 0.5 \mathrm{~K}$ with an electronically thermostatting single cell and monitored with thermocouples attached to the cell. The room temperature chemiluminescent fluorescence (RTCF) and the room temperature chemiluminescent phosphorescence thermal decay of the compounds (1a-3a) were determined using a photomultiplier tube. 


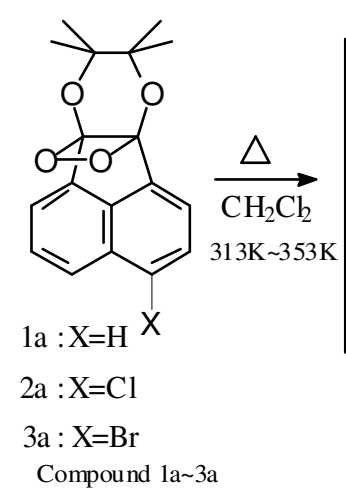

$3 \mathrm{a}: \mathrm{X}=\mathrm{Br}$

Compound 1a 3a

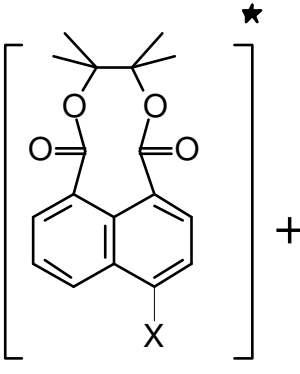

$\left(S_{1}\right)$<smiles>[X]c1cccc2c(C(=O)OCC)cccc12</smiles>

$\left(\mathrm{T}_{1}\right)$

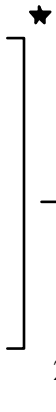

$2 \mathrm{~b}: \mathrm{X}=\mathrm{Cl}$ $3 b: \mathrm{X}=\mathrm{Br}$

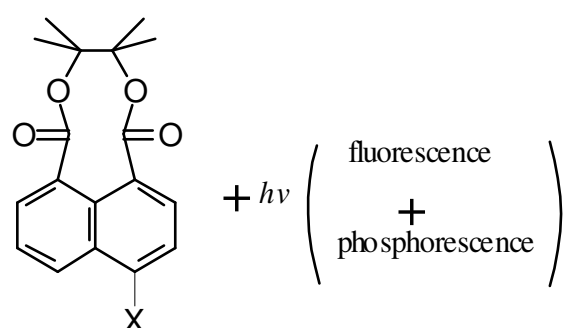

$\left(\mathrm{S}_{0}\right)$

Compound $1 b^{\star} \sim 3 b^{\star}$

Scheme-IV

\section{RESULTS AND DISCUSSION}

The isolated compounds (1a-3a) were formed by reacting compounds (1-3) with singlet-oxygen $\left({ }^{1} \mathrm{O}_{2}\right)$ in dichloromethane at $238 \mathrm{~K}$. Thermal decomposition of the 1,2-dioxetanes produce the electronically excited diesters $\left(\mathbf{1} \mathbf{b}^{*} \mathbf{- 3} \mathbf{b} *\right)$ that decayed by chemiluminescent fluorescence or chemiluminescent phosphorescednce to the ground state compounds (1b-3b). To avoid triplet-oxygen $\left({ }^{3} \mathrm{O}_{2}\right)$ quenching of the triplet excited states, we used the freeze-pump-thaw method to degas solutions where the compounds (1b-3b) are dissolved. Experimental evidences show that the chemiluminescence spectral peaks of the compounds (1a-3a) are consistent with that of the photoluminescence spectral peaks of the photoexcited nondegassed and degassed compounds (1b-3b). These spectroscopic observations suggest that the compounds (1a-3a) thermally decomposed to the excited state of the compounds $\left(\mathbf{1} \mathbf{b}^{*}-\mathbf{3} \mathbf{b}^{*}\right)$, with subsequent radiative decay to the ground state of the compounds (1b-3b). These processes are showed in Scheme-IV.

As an example, the UV/visible absorption and photoluminescence spectra of the compound $\mathbf{3 b}$ and the chemiluminescence spectrum of the compound $\mathbf{3 a}$ in dichloromethane are shown in Fig. 1. To compare the three spectra (chemiluminescence, nondegassed and degassed photoluminescence) conveniently, the chemiluminescence and photoluminescence spectral peaks at $\lambda_{\max }$ are normalized to same relative intensity. The experimental results show that the room temperature chemiluminescent fluorescence spectral peak of the compound 3a at $378 \mathrm{~nm}$ is consistent with that of the photoluminescence spectral peaks of the photoexcited nondegassed and degassed compound $\mathbf{3 b}$. The room temperature chemiluminescent phosphorescence spectral peak of the compound 3a at 560 $\mathrm{nm}$ is consistent with that of the photoluminescence spectral peak of the photoexcited degassed compound $\mathbf{3 b}$. The photoluminescence spectral peak at $560 \mathrm{~nm}$ of the photoexcited nondegassed compound $\mathbf{3 b}$ is not observed due to quenching of the triplet states by triplet-oxygen $\left({ }^{3} \mathrm{O}_{2}\right)$. The lifetimes of the two peaks in the chemiluminescence spectrum of the compound 3a were measured by monitoring the decays at fixed wavelength. The experimental results show the fluorescence and phosphorescence decay lifetimes of the degassed compound $\mathbf{3 b}$ in dichloromethane are 5.3 and $80 \mu \mathrm{s}$, respectively. The chemiluminescence spectral peaks are composed of chemiluminescent fluorescence and chemiluminescent phosphorescence (Fig. 1).

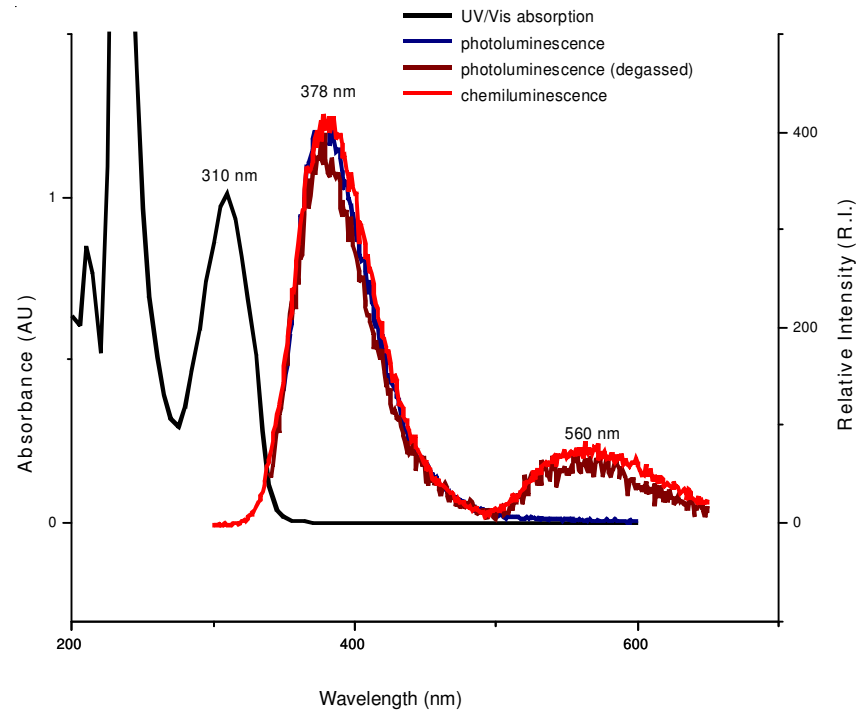

Fig. 1. UV/visible absorption, photoluminescence spectra of the photoexcited compound $\mathbf{3 b}$ and the room temperature chemiluminescent fluorescence and room temperature chemiluminescent phosphorescence spectra of the compound $\mathbf{3 a}$ at $343 \mathrm{~K}$ in dichloromethane

We observed quite different room temperature chemiluminescent phosphorescence spectra relative intensity between the nondegassed compounds $\mathbf{1 a}(\mathbf{H})-\mathbf{2} \mathbf{a}(\mathbf{C l})-\mathbf{3 a}(\mathbf{B r})$. It is known that the rate of a spin-forbidden process is enhanced by the presence of an atom of high atomic number. This factor is observed with the room temperature chemiluminescent phosphorescence. The 5-heavy atom substituted acenaphtho moiety of 1,2-dioxetanes shows enhanced nonradiative intersystem crossing to the lowest triplet state, resulting in an increase in the intensity of the phosphorescence. This increase of the room temperature chemiluminescent phosphorescence spectra relative intensity from triplet excited state due to the internal heavy atom substituted is $\mathrm{H}<\mathrm{Cl}<\mathrm{Br}$ (Fig. 2).

The photoluminescence spectral peaks of the photoexcited compounds (1b-3b) are little shifted toward longer wavelengths as the solvent is changed from the less polar dichloromethane to more polar acetonitrile. The room temperature chemiluminescent fluorescence and room temperature chemiluminescent phosphorescence derive from singlet and triplet $* \pi \rightarrow \pi$ states. An external heavy atom effect was observed with compound 3b in going from benzene to dichloromethane (Fig. 3). The intensity of phosphorescence of compound $\mathbf{3 b}$ which dissolved in dichloromethane is higher than that dissolved in benzene. 


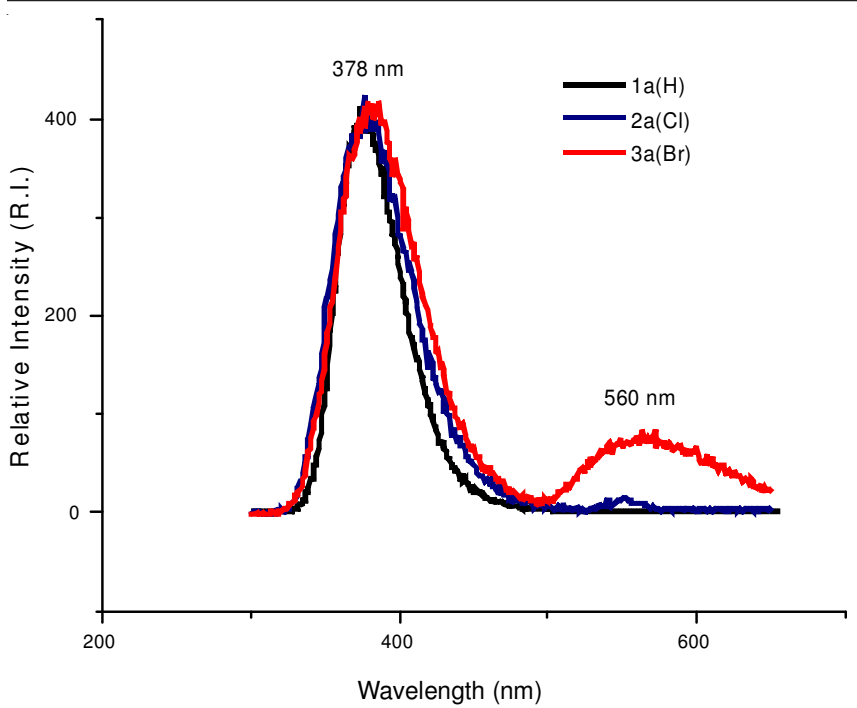

Fig. 2. Chemiluminescence of the compound $\mathbf{1 a}(\mathbf{H})-\mathbf{2} \mathbf{a}(\mathbf{C l})-\mathbf{3 a}(\mathbf{B r})$ at 343 $\mathrm{K}$ in dichloromethane

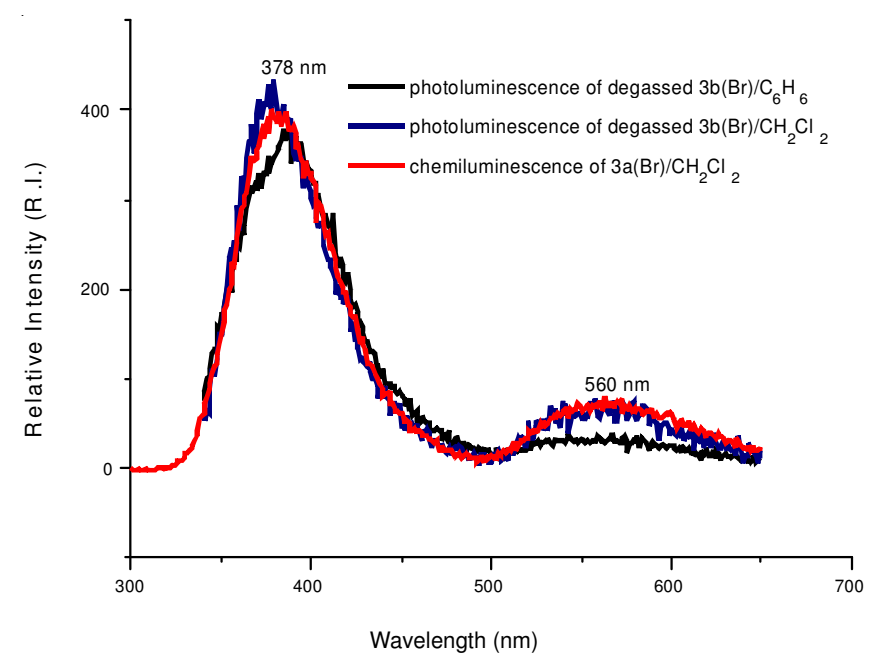

Fig. 3. Photoluminescence spectral peaks of the compound $\mathbf{3 b}$

The ratio $\left(\mathrm{RI}_{\lambda 560 \mathrm{~nm}} / \mathrm{RI}_{\lambda 378 \mathrm{~nm}}\right)$ of the degassed compound $\mathbf{3 b}$ in benzene solvent is 0.10 , while this ratio increases to 0.17 in dichloromethane solvent.

The rate constants for the decomposition of the compound 3a was obtained at $10 \mathrm{~K}$ increments between $313-353 \mathrm{~K}$ by measuring the decay of the chemiluminescence intensity at the maximum wavelength of the chemiluminescence in dichloromethane $\left(\lambda_{\mathrm{CF}, \max }: 378 \mathrm{~nm}, \lambda_{\mathrm{CP}, \max }: 560 \mathrm{~nm}\right)$. Fig. 4 show the chemiluminescent fluorescence and the chemiluminescent phosphorescence thermal decays of the compound 3a at $343 \mathrm{~K}$, monitored at 378 and $560 \mathrm{~nm}$, respectively.

The overall chemiluminescent mechanism of the 1,2dioxetane tricyclic compound $\mathbf{3 a}$ can be presented as eqn. 1.

$$
\mathrm{B} \stackrel{\frac{k_{1}}{k_{k}}}{\mathrm{X}^{\ddagger}} \rightarrow \mathrm{C}^{*} \rightarrow \mathrm{C}
$$

where $\mathrm{B}=1$,2-dioxetane tricyclic compound $\mathbf{3 a} ; \mathrm{X}^{\ddagger}=$ the activated complex; $\mathrm{C}^{*}=$ excited intramolecular diester compound $\mathbf{3 b}$.

From the Boltzmann distribution eqn. 2

$$
k=\left(\frac{k_{B} T}{h}\right) e^{\left(\frac{-\Delta G^{\ddagger}}{R T}\right)}
$$

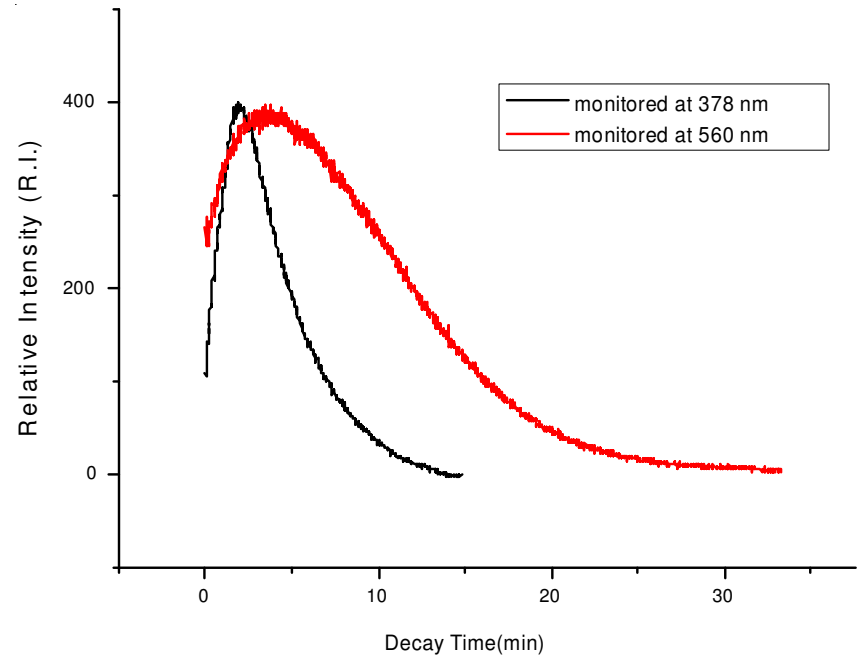

Fig. 4. Chemiluminescent fluorescence and chemiluminescent phosphorescence decay of the compound $\mathbf{3 a}$ at $343 \mathrm{~K}$

where $\mathrm{k}_{\mathrm{B}}=$ the Boltzmann constant; $\mathrm{h}=$ Planck's constant, $\Delta \mathrm{G}^{\ddagger}=$ the activation free energy of the transition state; $\mathrm{R}=$ the gas constant. The Gibbs free energy eqn. 3

$$
\Delta \mathrm{G}^{\ddagger}=\Delta \mathrm{H}^{\ddagger}-\mathrm{T} \Delta \mathrm{S}^{\ddagger}
$$

where $\Delta \mathrm{H}^{\ddagger}=$ the activation enthalpy of the transition state; $\Delta \mathrm{S}^{\ddagger}=$ the activation entropy of the transition state, is obtained by the Eyring eqn. 4 using transition-state theory ${ }^{13-15}$.

$$
\ln \frac{\mathrm{k}}{\mathrm{T}}=\left(\ln \frac{\mathrm{R}}{\mathrm{N}_{\mathrm{A}} \mathrm{h}}+\frac{\Delta \mathrm{S}^{\ddagger}}{\mathrm{R}}\right)-\frac{\Delta \mathrm{H}^{\ddagger}}{\mathrm{RT}}
$$

where $\mathrm{N}_{\mathrm{A}}=$ Avogadro's constant $\mathrm{h}=$ Planck's constant.

This equation relates $\ln (\mathrm{k} / \mathrm{T})$ and $(1 / \mathrm{T})$ as a linear function. A plot of $\ln (\mathrm{k} / \mathrm{T})$ versus $(1 / \mathrm{T})$ gives a straight line with slope $\left(-\Delta H^{\sharp} / \mathrm{R}\right)$ from which the activation enthalpy of the transition state may be derived. The chemiluminescent fluorescence and chemiluminescent phosphorescence decays from 1,2dioxetane 3a was analyzed by eqn. 4 . There are two slopes

$\left(\frac{\Delta \mathrm{H}_{\text {Singlet }}^{\ddagger}}{\mathrm{R}}\right),\left(\frac{\Delta \mathrm{H}_{\text {Triplet }}^{\ddagger}}{\mathrm{R}}\right)$ of the Eyring plots corresponding to the chemiluminescent fluorescence decay of compound $\mathbf{3 a}$ at $378 \mathrm{~nm}$ and the chemiluminescent phosphorescence decay of compound 3a at $560 \mathrm{~nm}$, respectively, as shown in Fig. 5. The activation parameters and rates of decomposition for compound 3a are listed in Table-1.

\begin{tabular}{ccccc}
\multicolumn{5}{c}{ TABLE-1 } \\
\multicolumn{5}{c}{$\begin{array}{c}\text { ACTIVATION PARAMETERS AND RATES OF } \\
\text { DECOMPOSITION FOR COMPOUND 3a }\end{array}$} \\
\hline $\begin{array}{ccccc}\lambda_{\max }(\mathrm{nm}) \\
(\mathrm{kcal} / \mathrm{mol})\end{array}$ & $\begin{array}{c}\Delta \mathrm{H}^{\ddagger} \\
(\mathrm{kcal} / \mathrm{mol})\end{array}$ & $\begin{array}{c}\Delta \mathrm{S}^{\ddagger} \\
(\mathrm{cal} / \mathrm{mol} \mathrm{K})\end{array}$ & $\begin{array}{c}\Delta \mathrm{G}^{\ddagger}\left(25^{\circ} \mathrm{C}\right) \\
(\mathrm{kcal} / \mathrm{mol})\end{array}$ & $\begin{array}{c}\mathrm{k}_{25}{ }^{\circ} \mathrm{C} \\
\left(\mathrm{s}^{-1}\right)\end{array}$ \\
\hline $378(75.7)$ & 22.2 & -5.1 & 23.7 & $2.44 \times 10^{5}$ \\
$560(51.1)$ & 15.2 & -26.5 & 23.1 & $7.6 \times 10^{-5}$ \\
\hline
\end{tabular}

The results show that there are two transition excited energy states in the chemiexcitation processes: (1) The transition singlet excited state activation enthalpy $\left(\Delta \mathrm{H}_{\text {Singlet }}^{\ddagger}\right)$. (2) The transition triplet excited state activation enthalpy 


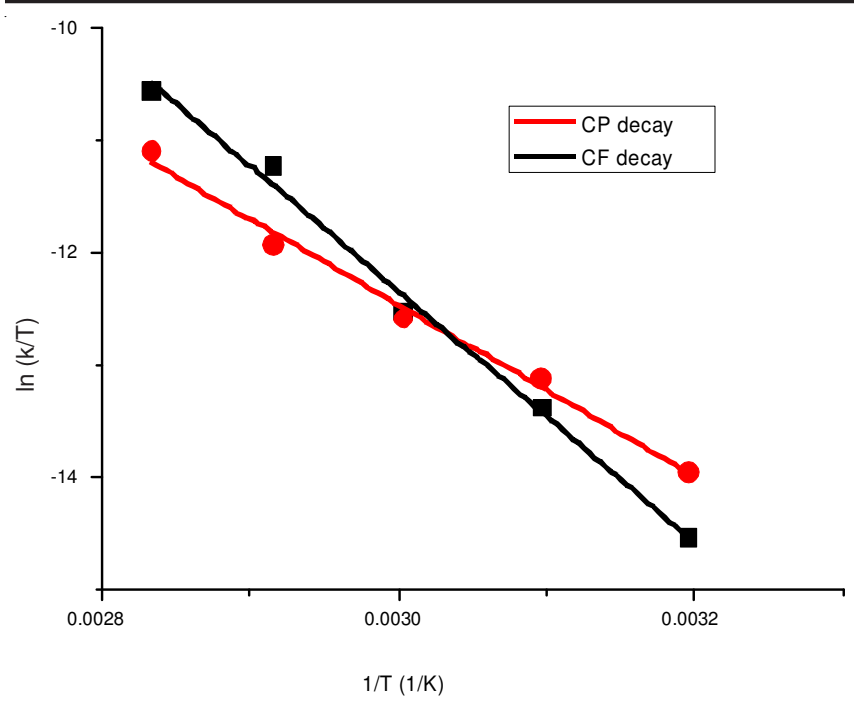

Fig. 5. Eyring plots of the chemiluminescence kinetics of compound 3a

$\left(\Delta \mathrm{H}_{\text {Triplet }}^{\ddagger}\right)$. The singlet excited state activation enthalpy $\left(\Delta \mathrm{H}_{\text {Singlet }}^{\ddagger}\right)$ of compound $\mathbf{3 a}$ has been determined experimentally to be $22.2 \mathrm{kcal} / \mathrm{mol}$. The triplet excited state activation enthalpy $\left(\Delta \mathrm{H}_{\text {Triplet }}^{\ddagger}\right)$ of compound 3a has been determined experimentally to be $15.2 \mathrm{kcal} / \mathrm{mol}$. The different activation enthalpies show the chemiluminescent phosphorescence and chemiluminescent fluorescence arise by competing path in the decomposition of the dioxetane and the lower enthalpy of activation results from the lower enthalpy (greater stability) of the lower lying triplet state. The rate constant of the triplet state obtained at $298 \mathrm{~K}$ is also higher than that of the singlet state. The different activation enthalpies and different rate constants seem to be a reasonable explanation that the triplet state is not formed by way of the singlet state and the chemiexcitation processes favour the pathway for the formation of the chemiluminescent phosphorescence.

The rate of a spin-forbidden process is enhanced by the internal presence of bromo-substituent, result the increase of the relative intensity of the room temperature chemiluminescent phosphorescence of compound 3a. The enhancement of the phosphorescence of compound $\mathbf{3 b}$ is also observed by the external heavy atom solvent. The calculations of the room temperature chemiluminescent fluorescence and the room temperature chemiluminescent phosphorescence decay time at variable temperature obtain the activation parameters for compound 3a. The enthalpy $\left(\Delta \mathrm{H}^{0}\right)$ of reaction for thermal decompositions of typical dioxetanes have been estimated from thermal chemical calculations from 69 to $90 \mathrm{kcal} / \mathrm{mol}^{16}$. The energy relationship diagram of the compound $\mathbf{3 a}$ thermal decomposed to compound $\mathbf{3 b}$ can be sketched, if we postulate the enthalpy of reaction for thermal decomposition of compound 3a symbol for " $\Delta \mathrm{H}^{0}$ ". The energy relationship diagram is showed in Scheme-V.

\section{Conclusion}

The unique feature of this work lies in the observed room temperature chemiluminescent phosphorescence of the 5-heavy atom substituted acenaphtho lumiphor. In the 1,2dioxetane ring of compound $\mathbf{3 a}$ which has high strain energy

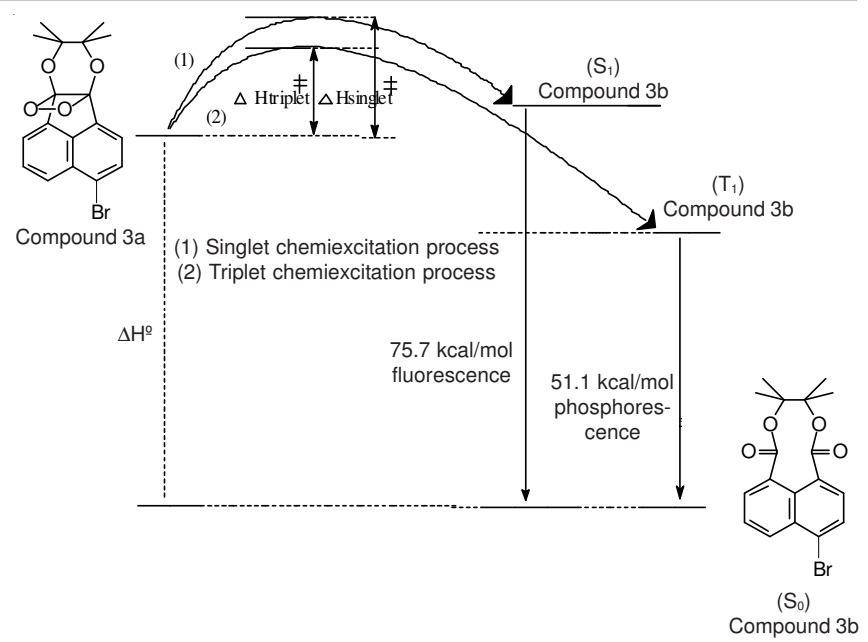

Scheme-V

due to the twisted four-membered ring peroxide structure (C-O-O-C), both the O-O and C-C bonds are cleaved almost simultaneously during thermal decomposition into two excited pathways: (1) singlet chemiexcitation processes, (2) triplet chemiexcitation processes. The internal heavy atom effect of 5-heavy atom substituted is $\mathrm{Br}>>\mathrm{Cl}>\mathrm{H}$. The transition triplet excited state activation enthalpy $\left(\Delta \mathrm{H}_{\text {Triplet }}^{\ddagger}\right)$ is lower than the transition singlet excited state activation enthalpy $\left(\Delta \mathrm{H}_{\text {Singlet }}^{\ddagger}\right)$ ca. $7 \mathrm{kcal} / \mathrm{mol}\left(\Delta \mathrm{H}_{\text {Triplet }}^{\ddagger}<\Delta \mathrm{H}_{\text {Singlet }}^{\ddagger}\right)$. We have shown that the 1,2-dioxetane tricyclic compound-based chemiluminescence especially chemiluminescent phosphorescence of compound 3a. We continue to investigate these and other aspects, example as 5-iodo substituted 1,2-dioxetane tricyclic compound.

\section{ACKNOWLEDGEMENTS}

Financial support from the National Science Council, Ming Chi University of Technology and National Taiwan Normal University, Taiwan, Republic of China, is gratefully acknowledged. Prof. L.A. Singer's (USC) comment is also highly appreciated.

\section{REFERENCES}

1. L.D. Vico, Y.-J. Lin, J.W. Krogh and R. Lindh, J. Phys. Chem. A, 111, 8013 (2007).

2. H. Isobe, Y. Takano, M. Okumura, S. Kuramitsu and K. Yamaguchi, $J$. Am. Chem. Soc., 127, 8667 (2005).

3. W. Adam, D.V. Kazakov and V.P. Kazakov, Chem. Rev., 105, 3371 (2005).

4. M. Matsumoto, J. Photochem. Photobiol. C, 5, 27 (2004).

5. G.B. Schuster, Acc. Chem. Res., 12, 366 (1979).

6. C. Tanaka, J. Tanaka and M. Matsumoto, Phys. Chem. Chem. Phys., 13, 16005 (2011)

7. H.S. Hewage, K.J. Wallace and E.V. Anslyn, Chem. Commun., 3909 (2007).

8. W. Adam, I. Bronstein and A.V. Trofimov, J. Phys. Chem. A, 102, 5406 (1998).

9. S.M. Silva, K. Wagner, D. Weiss, R. Beckert, C.V. Stevani and W. Baader, J. Luminescence, 17, 362 (2002).

10. L. Wang, X. Wang, J. Cui, W. Ren, N. Meng, J. Wang and X. Qian, Tetrahedron: Asym., 21, 825 (1987).

11. T.-S. Fang and W.-P. Mei, Tetrahedron Lett., 28, 329 (1987).

12. T.-S. Fang and L.A. Singer, J. Am. Chem. Soc., 100, 6276 (1978).

13. H. Eyring, J. Chem. Phys., 3, 107 (1934).

14. K.J. Laldler and M.C. King, J. Phys. Chem., 87, 2657 (1983).

15. D.J. Winzor and C.M. Jackson, J. Mol. Recognit., 19, 389 (2006).

16. W.H. Richardson and H.E. O'Neal, J. Am. Chem. Soc., 94, 8665 (1987). 